\title{
OPEN Stimulus blanking reveals contrast-dependent transsaccadic feature transfer
}

\author{
Lukasz Grzeczkowski ${ }^{1}{ }^{凶}$, Heiner Deubel ${ }^{1}$ \& Martin Szinte ${ }^{2,3}$
}

Across saccadic eye movements, the visual system receives two successive static images corresponding to the pre- and the postsaccadic projections of the visual field on the retina. The existence of a mechanism integrating the content of these images is today still a matter of debate. Here, we studied the transfer of a visual feature across saccades using a blanking paradigm. Participants moved their eyes to a peripheral grating and discriminated a change in its orientation occurring during the eye movement. The grating was either constantly on the screen or briefly blanked during and after the saccade. Moreover, it either was of the same luminance as the background (i.e., isoluminant) or anisoluminant with respect to it. We found that for anisoluminant gratings, the orientation discrimination across saccades was improved when a blank followed the onset of the eye movement. Such effect was however abolished with isoluminant gratings. Additionally, performance was also improved when an anisoluminant grating presented before the saccade was followed by an isoluminant one. These results demonstrate that a detailed representation of the presaccadic image was transferred across saccades allowing participants to perform better on the transsaccadic orientation task. While such a transfer of visual orientation across saccade is masked in real-life anisoluminant conditions, the use of a blank and of an isoluminant postsaccadic grating allowed to reveal its existence.

The mosaic of photoreceptors of the human retina is highly inhomogeneous ${ }^{1}$. This results in high resolution vision in the center of the visual field and low resolution vision in its periphery ${ }^{2}$. To account for that inhomogeneity, humans sample their environment by making frequent saccadic eye movements. But to make a saccade to an object of interest, the visual system must first select that object in between others. This selection is necessarily based on the low-resolution information available in the visual periphery that is replaced by the high resolution, foveal image at the end of the saccade ${ }^{3}$. Thus, across saccades, the visual system receives two images of an object, a presaccadic low-resolution image in its periphery, and a postsaccadic high-resolution image in its fovea ${ }^{4}$. The mechanisms used by the visual system to account for these resolution changes is still today a matter of debate ${ }^{5}$. One mechanism used probably is the enhancement of visual sensitivity at the saccade target position just before the execution of a saccade ${ }^{3,6,7}$. However, an open question resides on whether the visual content of the presaccadic image can be transferred across saccades and to which extent it is integrated with the postsaccadic image ${ }^{8,9}$.

Most of the early studies on that question concluded that transsaccadic perception relies on visual short term memory with a transfer of abstract, relational and structural aspects of visual stimuli across saccades ${ }^{10}$. Moreover, it was proposed that the detailed presaccadic information, the stimulus features and its precise position are not transferred, nor integrated across saccades ${ }^{11-14}$. Recent studies however changed this view, showing that low level, pre- and postsaccadic image visual features such as orientation ${ }^{15-17} \operatorname{color}^{18-20}$ or spatial frequency ${ }^{21}$ are indeed partially transferred and integrated across the saccade, probably through remapping mechanisms ${ }^{22-26}$. Importantly for the context of the present study, it was also shown that relatively precise information of object location can be maintained across saccades and be almost perfectly recovered if the attended object is briefly blanked at the onset of the saccade ${ }^{27,28}$. Interestingly, this blanking effect turned out to be strongly reduced for low contrast and isoluminant stimuli ${ }^{29,30}$, suggesting that the mechanism used to transfer position across saccades relies on luminance contrast information. In line with the above findings, a recent study ${ }^{31}$ showed that two sets of lines presented before and after eye movements could be perceptually fused to form a letter if the postsaccadic lines are of low contrast.

\footnotetext{
${ }^{1}$ Allgemeine und Experimentelle Psychologie, Ludwig-Maximilians-Universität München, Leopoldstrasse 13, 80802 Munich, Germany. ${ }^{2}$ Institut de Neurosciences de la Timone, UMR 7289, Centre National de la Recherche Scientifique, Aix-Marseille Université, Marseille, France. ${ }^{3}$ Spinoza Centre for Neuroimaging, Royal Dutch Academy of Sciences, Amsterdam, The Netherlands. ${ }^{\circledR}$ email: lukasz.grzeczkowski@gmail.com
} 
A

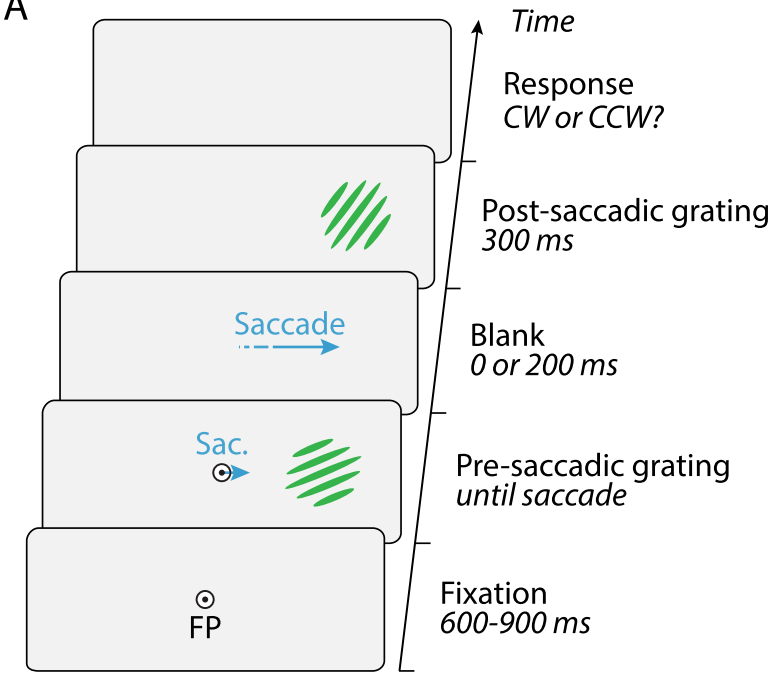

B
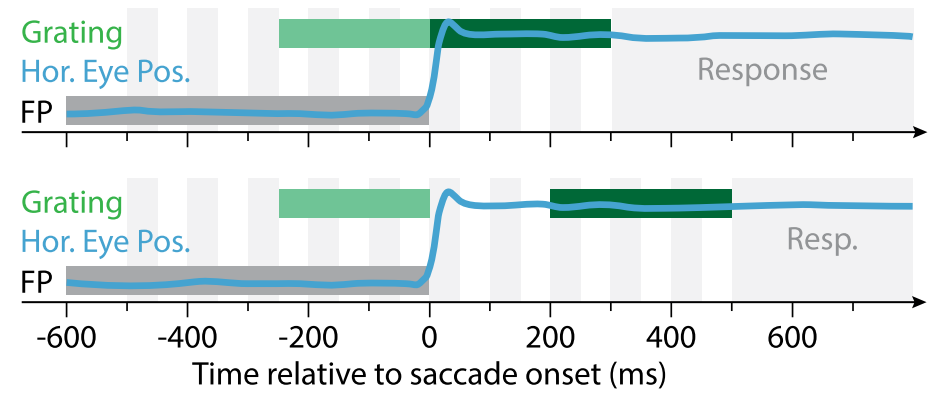

C

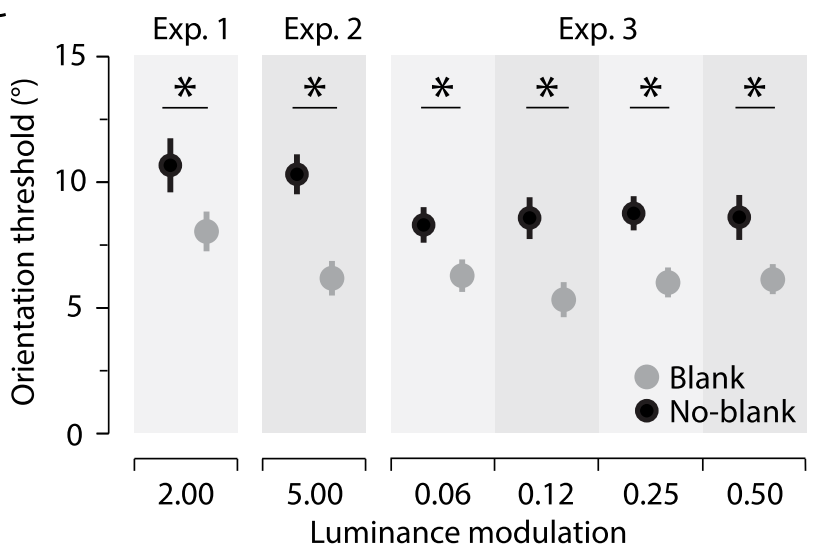

D

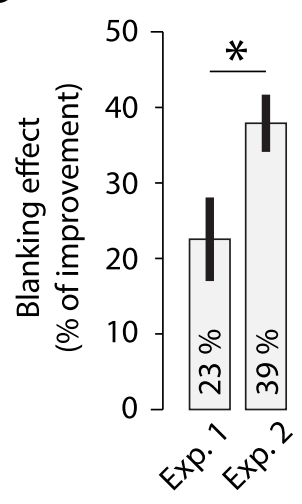

E

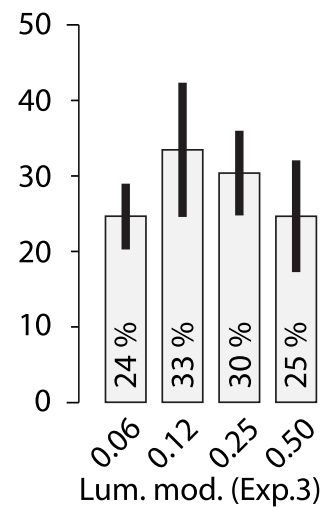

Figure 1. Procedure and orientation blanking effect. (A) In all experiments, after fixating the fixation point (FP), participants made a saccade towards a peripheral presaccadic oriented grating. The saccade triggered the presentation of the postsaccadic grating, rotated clockwise or counterclockwise relative to the presaccadic one. The postsaccadic grating was presented either immediately ( $0 \mathrm{~ms}$, No-blank condition) or $200 \mathrm{~ms}$ after a period during which the screen was blanked (Blank condition). Participants reported the direction of the orientation change at the end of each trial. (B) Time course of a typical No-blank and Blank trial (upper and lower panels, respectively). The blue line shows horizontal eye position (Hor. Eye Pos.) relative to saccade onset. Rectangles illustrate the timing of the pre- (light green) and postsaccadic (dark green) gratings and the fixation point (FP, gray). (C) Average orientation threshold angle observed with anisoluminant gratings in Exp.1, Exp. 2 and Exp. 3 , in the No-blank (black) and Blank (gray) conditions, respectively. (D,E) Bars show the blanking effect (see methods) observed in the Exp.1, Exp.2 and Exp. 3. Error bars show SEM and asterisks and lines show significant comparisons $(\mathrm{p}<0.05)$.

Here, we hypothesize that the blanking effect ${ }^{27}$ relies on the transfer across saccades of a remapped, representation of the presaccadic image. To test this hypothesis, we evaluated participants' ability to judge a change of a grating orientation occurring during a saccade (Fig. 1A,B). We predicted that the addition of a short blank during and after the saccade would help participants to judge the transsaccadic orientation change, as it was previously demonstrated for transsaccadic changes of spatial frequency ${ }^{21}$ and object detail ${ }^{32,33}$. We hypothesized that with a blank, participants could base their judgment on the memory trace of the transferred presaccadic orientation and the postsaccadic orientation. Moreover, given that the blanking effect for object location depends on the luminance contrast of the stimulus ${ }^{29}$, we predicted that only judgments made for anisoluminant stimuli would benefit from the blank. Our results confirmed these hypotheses, demonstrating a strong improvement of discrimination thresholds when a blank was introduced at the saccade onset. Moreover, detailed recovery of the presaccadic object orientation across saccades was observed only for anisoluminant, but not for isoluminant stimuli. Remarkably, in the No-blank condition, performance was also improved when presaccadic anisoluminant gratings were followed by isoluminant postsaccadic gratings.

Together, these results suggest the existence of a mechanism allowing the transfer and the integration of visual features across saccades that strongly relies on the luminance contrast. We here made such a mechanism directly apparent by the use of a short blank during and after the eye movement. 
A

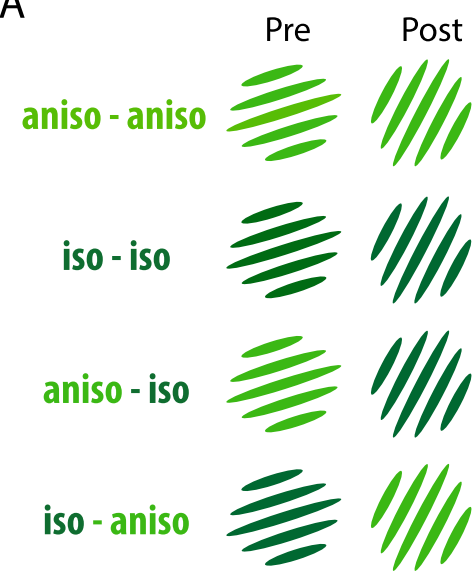

B

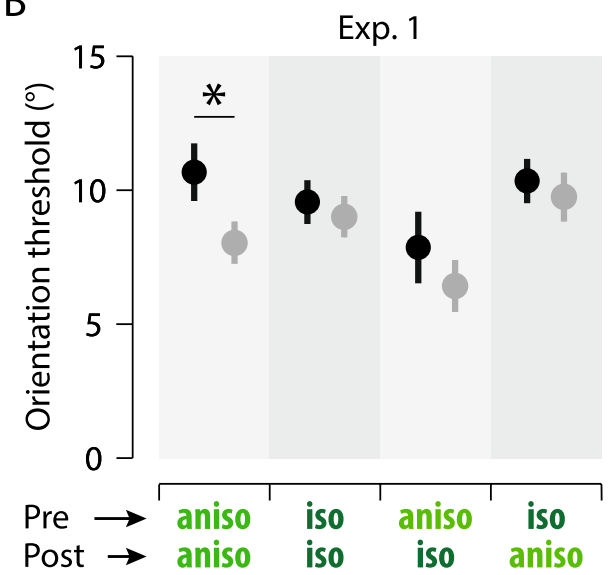

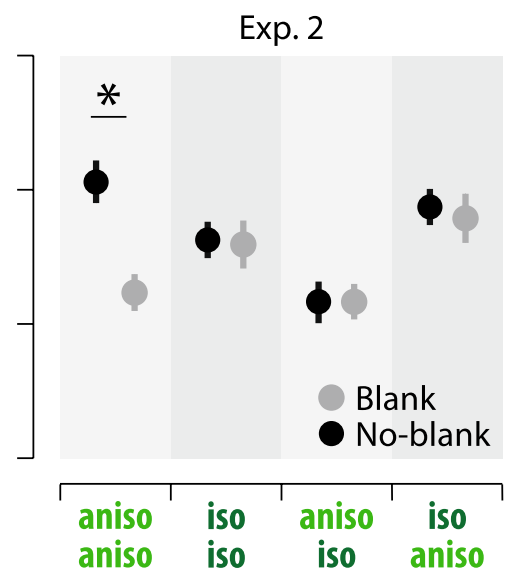

Figure 2. Effect of luminance on the orientation blanking effect. (A) Combinations of pre- and postsaccadic gratings used in Exp. 1 and Exp. 2. (B) Mean thresholds observed as a function of the pre- and postsaccadic grating luminance, in the No-blank (black) and blank (gray) conditions, respectively. Note that results with preand postsaccadic anisoluminant gratings are here replotted from Fig. 1. Conventions are as in Fig. 1.

\section{Results}

In Exp. 1 and Exp. 2, we tested four combinations of iso- and anisoluminant gratings at the pre- and postsaccadic locations. In Exp. 3 we tested only anisoluminant gratings in four different luminance conditions. For the sake of simplicity, we will first report the results for the anisoluminant, "natural" conditions and then turn to the isoluminant conditions of Exp. 1 and Exp. 2.

We first determined how large the change of the grating orientation must be such that participants can correctly discriminate its orientation change across saccades. We found that with anisoluminant gratings, that is gratings with a twofold (Exp. 1) or fivefold (Exp.2) luminance relative to the background, participants needed a rotation change of about $10.5^{\circ}$ relative to presaccadic grating angle to discriminate the change correctly in $75 \%$

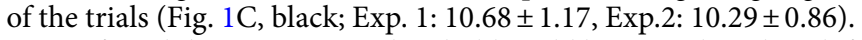

We found that orientation threshold could be strongly reduced if a $200 \mathrm{~ms}$ blank followed the saccade onset. When a blank was used, participants needed only a rotation of $\sim 7^{\circ}$ to perform the task (Fig. 1C, gray; Exp. 1: $8.05 \pm 0.85, p=0.0381, d=0.81$; Exp. $2: 6.17 \pm 0.52, p<0.0001, d=1.84)$. On average, orientation discrimination thresholds were improved by about $30 \%$ with the use of a blank, an improvement slightly less pronounced with twofold (Fig. 1D, Exp. 1: $23.20 \pm 5.73 \%$ ) than with fivefold luminance gratings (Fig. 1D, Exp. 2: $38.80 \pm 3.91 \%$, $p=0.0172, d=0.92$ ). Interestingly, as a blank is by definition an absence of visual stimulation, these first results suggest that the transfer of the presaccadic orientation is partially masked by the presentation of the postsaccadic grating without a blank. In other words, the blank allows to recover better the feature content of the grating orientation across saccades by unmasking the presaccadic transferred information.

In Exp. 3, we tested whether the blanking effect occurs for much less luminant gratings, i.e., gratings having $6,12,25$ and $50 \%$ more luminance than the background. We found the orientation blanking effect at each of these luminance conditions, with significant improvement of the orientation threshold within the similar range as in the Exps. 1 and 2 (Fig. 1C-E; 0.0265 $>p s>0.0013,1.63>d s>0.86$ ). These results suggest that the orientation blanking effect is a robust phenomenon which reaches a plateau already at $\sim 6 \%$ of luminance with respect to the background.

While we systematically found an orientation blanking effect with anisoluminant gratings, we also tested the three other conditions in which we varied the luminance of the pre- and postsaccadic gratings (Fig. 2). We will now focus on the results concerning the isoluminant gratings from Exp. 1 and Exp. 2. First, when both the pre- and postsaccadic gratings were isoluminant, i.e., gratings only defined by a change in color relative to the background, there was no difference in orientation threshold when comparing trials with a blank (Fig. 2B: presac iso and post-sac iso, Exp. 1: $9.01 \pm 0.83$; Exp. 2: 7.96 \pm 0.97 ) and without it (Fig. 2B: pre-sac iso and post-sac iso, Exp. 1: 9.56 $\pm 0.89, p=0.62, \mathrm{~d}=0.20$; Exp. 2: $8.13 \pm 0.77, p=0.88, d=0.06$ ). Importantly, these results show that unmasking the transferred targets representation by blanking has no effect. This suggests that the precise representation of the presaccadic grating is only available after the saccade if the presaccadic image is defined by luminance contrast ${ }^{29}$.

Second, no blanking benefits were found in trials in which a presaccadic isoluminant grating was followed by a postsaccadic anisoluminant one. In this condition, there was no improvement of orientation discrimination threshold when comparing trials with (Fig. 2B: pre-sac iso and post-sac aniso, Exp. 1: 9.75 \pm 1.00 ; Exp. 2: $8.93 \pm 1.00$ ) and without a blank (Fig. $2 B$ : pre-sac iso and post-sac aniso, Exp. 1: $10.91 \pm 0.91, p=0.61, d=0.30$; Exp. 2: $9.36 \pm 0.63, p=0.67, d=0.21$ ). This result further demonstrates that, similarly to the previous condition where pre- and postsaccadic gratings were both isoluminant, the transsaccadic transfer of orientation depends on the luminance contrast of the presaccadic image.

Finally, we tested a condition in which a presaccadic anisoluminant grating was followed by a postsaccadic isoluminant one. Here again, we didn't find a blanking effect when comparing trials with (Fig. 2B: pre-sac aniso and post-sac iso, Exp. 1: 6.44 \pm 1.10; Exp. 2: 5.83 \pm 0.61 ) and without a blank (Fig. 2B: pre-sac aniso and post-sac 
A

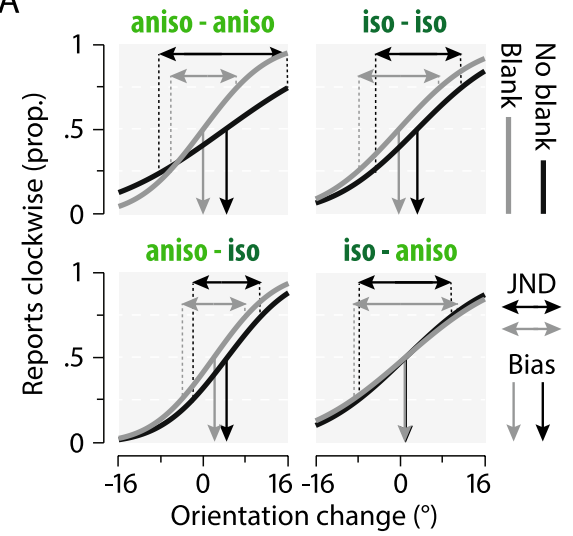

B

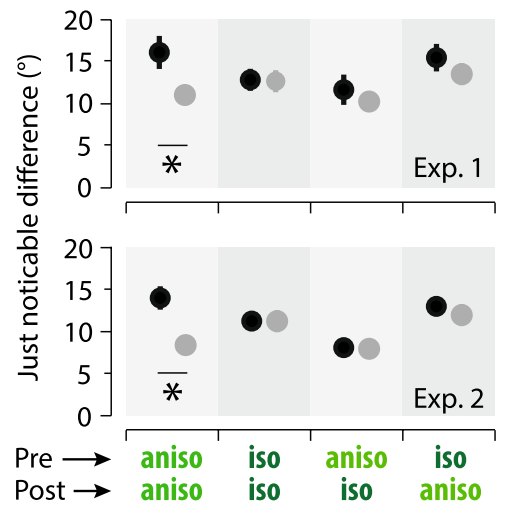

C

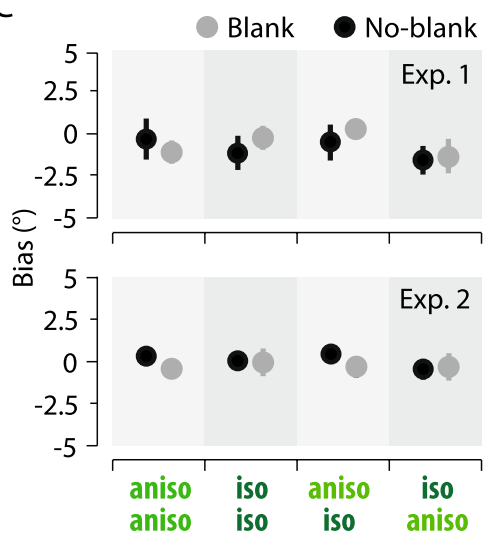

Figure 3. Just noticeable differences (JNDs) and biases as a function of the gratings' luminance. (A) Psychometric functions of a representative participant showing the proportion of clockwise responses as a function of the orientation change of the gratings across saccade in each luminance (see panel titles) and blanking condition (Blank and No-blank in gray and black, respectively). Horizontal and vertical arrows show JNDs and biases, respectively for the blank (gray) and No-blank (black) conditions. Negative angle values correspond to counterclockwise angle changes. (B,C) Average observed JNDs (B) and biases $(\mathbf{C})$ across participants in Exp. 1. (upper panels) and Exp. 2 (lower panels). Conventions are as in Fig. 1.

iso, Exp. 1: threshold $=7.87 \pm 1.46, p=0.36, d=0.35$; Exp. 2 : threshold $=5.80 \pm 0.55, p=0.95, d=0.02$ ). One could first conclude that also in this condition, a precise stimulus memory trace was not available to the participants after their saccade. However, orientation thresholds in this condition (pre-sac aniso and post-sac iso) were systematically lower than those observed with anisoluminant pre- and postsaccadic gratings in both experiments (Exp. 1: $10.68 \pm 1.17$ vs $6.44 \pm 1.10$; Exp. $2: 10.29 \pm 0.86$ vs $5.83 \pm 0.61$ ). The difference between these two conditions was significant in Exp.2 (Fig. 2B; Exp. 2, $p<0.0001, d=1.98)$ but not in Exp. $1(p=0.0943, d=0.67)$, with a Bayes factor however strongly supporting the rejection of the null hypothesis for that comparison $\left(\mathrm{BF}_{10}=11.12\right)$. This pattern of results may suggest that a precise memory trace of the presaccadic grating is present after the saccade when an object defined by luminance contrast (but not color) is presented as a saccade goal. This transsaccadic image is normally masked by the content of the postsaccadic image, at least when this second image contains luminance contrast. However, in the aniso-iso condition the postsaccadic stimulus does not contain luminance contrast and is therefore unable to mask the presaccadic memory trace. Similarly to the aniso-aniso condition with a blank, the presaccadic memory trace may be not masked which results in better performance.

How precise is the transferred representation? To answer this question, we analyzed participants' just noticeable differences (JND) and biases in discriminating the orientation change (see "Materials and methods" and Fig. 3). We found large JND improvements when both, the pre- and postsaccadic gratings were anisoluminant as compared to other conditions. This effect is made evident for a representative participant (Fig. 3A, top-left panel), showing a steeper psychometric function in the Blank (gray symbols) than in the No-blank (black symbols) condition. This led to significant reductions of the observed JNDs when both, the pre- and the postsaccadic gratings were anisoluminant, for the No-blank (Fig. 3B; Exp. 1: JND =16.25 \pm 1.95 ; Exp. 2: 12.96 \pm 1.34 ) and Blank conditions (Exp. 1: JND = 10.96 $\pm 0.99, p<0.0121$; Exp. 2: $8.15 \pm 0.63, p=0.0007$ ). Interestingly, such improvement of discrimination performance was made apparent by the use of blank without change of participant judgment bias when comparing the No-blank (Fig. 3C; Exp. 1: Bias $=-0.21 \pm 1.25$; Exp. 2: Bias $=0.33 \pm 0.62$ ) and the Blank condition (Exp. 1: Bias $=-1.01 \pm 0.71, p=0.57$; Exp. 2: Bias $=-0.42 \pm 0.41, \mathrm{p}=0.29$ ). These results contrasted with what observed within our three other luminance conditions, for which we found that both the participants' JNDs (Fig. 3B; Exp. 1: 0.84>ps>0.33; Exp. 2: 0.94>ps>0.59) and biases (Fig. 3C; Exp. 1, $0.83>p s>0.45$; Exp. 2, $0.87>p s>0.29$ ) stayed constant when comparing the No-blank and the Blank conditions. Together these results suggest that the transferred representation is more precise than indicated by the normal, No-blank condition. When available, it rendered the orientation discrimination task easier over the whole range of the tested orientation changes without affecting participant bias.

\section{Discussion}

Here, we found that visual orientation information is partly transferred across saccades, as reported earlier ${ }^{15-17}$. This feature transfer allows participants to report the orientation change of a grating across eye movements. Importantly, we showed in three experiments that briefly blanking the screen during and after the saccade allowed participants to recover the orientation of the presaccadic grating, as made evident by the strong and systematic improvement of participants' orientation thresholds and JNDs. Moreover, while we found the strongest orientation blanking effect with gratings of high luminance (Exps. 1 and 2), the effect seemed to reach its maximum with low luminance stimuli already (Exp. 3: between 6 and 12\% more luminance then the background), suggesting the generality of the underlying mechanism. These results differ from a previous report ${ }^{29}$ in which the blanking effect for position had a gradual effect as a function of the contrast level. Nevertheless, with orientation change discrimination, we similarly found an absence of a blanking effect for isoluminant stimuli ${ }^{29}$. This effect 
is particularly interesting as it suggests that feature transfer across saccades relies on the presence of luminance contrast in the presaccadic scene. Moreover, it demonstrates that the presence of a blank after saccade onset allows the recovery of presaccadic information that is usually masked by the postsaccadic image. In line with that result, participants better reported the orientation change when the presaccadic anisoluminant grating was followed by an isoluminant one, irrespectively of the presence of a blank. This suggests that the transferred orientation across saccades wasn't masked as effectively by a postsaccadic isoluminant grating as it was by an anisoluminant one. Finally, the changes in the just notifiable difference (JND) observed with anisoluminant gratings suggest that the transsaccadic judgment task was easier when a blank followed an anisoluminant grating (aniso-aniso, Fig. 3) or when the postsaccadic grating was isoluminant irrespectively of the presence of a blank (aniso-iso, Fig. 3).

Our results point towards the existence of a visual mechanism allowing the transfer of visual features across saccades. Indeed, our results suggest that the precise memory trace of the presaccadic object, normally masked by the postsaccadic object can be accessible to participants when a blank is used. We propose that this memory trace is accessible by the blank and induces a percept of apparent motion between the spatiotopic image of the presaccadic grating and the postsaccadic grating ${ }^{34}$, making the transsaccadic orientation discrimination task easier. Importantly, our results, together with previous ones showing a blanking effect for spatial frequency discrimination ${ }^{21}$ imply that low-level visual features are transferred across saccades and that under normal conditions, the transsaccadic memory is, to a large extent, hampered by the postsaccadic scene. In this framework, blanking the postsaccadic scene postpones or avoids the masking of the transferred visual feature enabling access to its content maintained in memory ${ }^{35}$.

It was recently shown that predictive coding can account for feature transfer across saccades ${ }^{36,37}$. Predictable objects were shown to be better detected ${ }^{36}$ and to lead to decreased BOLD activity in the early visual cortex as compared to unpredictable ones ${ }^{37}$. Predictive coding models assume that visual stimuli after being processed in high-level visual areas are fed back to low-level neurons in order to create visual predictions ${ }^{38}$. In a similar manner, high-level processes can generate low-level percepts through visual imagery. Visual imagery can lead to the formation of phantom-like percepts that can bias future perception ${ }^{39}$ and even lead to visual perceptual learning ${ }^{40}$. Besides visual imagery, phantom percepts such as observed in the filling-in phenomenon were shown to generate neural activity in the early visual corte ${ }^{41}$. We thus argue that, alike visual imagery and filling-in phenomena, we here demonstrated a phantom-like featural memory trace that could result from predictive coding fed back to low-level visual neurons. In line with this proposal, we show an increased blanking effect in Exp. 2 as compared to Exp. 1 and Exp. 3 that can be due to a stronger prediction of the upcoming stimulus and therefore a stronger phantom percept. Our results are in agreement with recent studies demonstrating the existence of a partial but systematic transsaccadic integration of visual features across saccades ${ }^{15-17,19,26,42}$ as well as the neural signature of the transsaccadic memory ${ }^{43,44}$.

We propose that the presaccadic stimulus first generates a feedforward propagation of the signal to higherlevel visual areas, then it is remapped and fed back to early visual areas at predicted spatiotopic locations. This back-propagation occurs during the saccade and ends after the saccade offset. Moreover, this predictive activation of low-level neurons, similarly to visual imagery or filling-in phenomena could create a phantom-like, featural memory trace. Interestingly, $50 \mathrm{~ms}$ after the saccade offset, the detection advantage of target predictability ${ }^{36}$ and the blanking effect for target displacement begin to be effective ${ }^{27}$.

To be seen at the correct position in space despite change in retinal coordinates, featural predictions must be remapped between the visual neurons accounting for the different pre- and the postsaccadic retinal positions of the gratings. A transfer of neural information of visual object position was demonstrated in higher level visual areas such as the $\mathrm{FEF}^{45}$, parietal areas ${ }^{24}$ and the superior colliculus ${ }^{46}$ which feeds back visual position information to low-level visual areas involved in visual orientation processing ${ }^{47}$. But to allow a spatiotopic representation of visual features, remapping of orientation information in our experiments must also take place. Up to date, such neural transfer of feature preference across saccades has been found in a small portion of tested neurons within for example the lateral intraparietal cortex ${ }^{48}$ and the middle temporal area $^{49}$, hardly explaining the strong behavioral effects. Possibly, however, the feedforward signals of the postsaccadic image, akin our masking effect observed with postsaccadic anisoluminant gratings, could have hampered the measure of the feature transfer. Future work using the blanking paradigm may demonstrate the existence of feature-specific, neural remapping mechanisms.

It was previously argued that only the position of attended objects would be transferred across the saccade ${ }^{5,8}$. However, our results as well as evidence of transsaccadic feature integration ${ }^{15,16}$ suggest that not only the position but also visual features are transferred. In this regard, others argued that head-centered, spatiotopic, or gazemodulated mechanisms could explain space constancy and transsaccadic perception ${ }^{9}$. While the visual system has been shown to be organized in retinotopic coordinates ${ }^{50}$, our results suggest that at one stage of the visual hierarchy the featural content of the presaccadic image is maintained in space. Thus, our results support the existence of a neural mechanism allowing visual neurons to keep track of the orientation of the grating across saccades. However, today, there is no clear available electrophysiological evidence favoring this alternative.

Irrespective of the neural mechanism behind, our results argue in favor of a precise, phantom-like, featural representation potentially ensuring space constancy. However, our results also suggest that under natural, anisoluminant conditions, such representation is partially masked, as also proposed previously ${ }^{51}$. While transsaccadic partial transfer of visual features might be sufficient for space constancy, it could also well be that feature information is generally not used across saccades or integrated with the postsaccadic information ${ }^{15,16}$. If that would be the case, maintaining the position of attended objects across saccade ${ }^{5,23}$ or assuming visual stability of the world could be sufficient to create an impression of stability. The transfer of visual features as observed here will then only reflect the fact that we experimentally broke this assumption using a blank.

Interestingly, one study showed that breaking transsaccadic object correspondence has similar effects as blanking in improving target location discrimination ${ }^{51}$. This study suggests that when the continuity of the target is disrupted, the pre- and the postsaccadic targets are processed as separate objects. Recently, a Bayesian causal 
inference model was proposed ${ }^{52}$ explaining the potential mechanisms underlying such processing. By establishing whether the transsaccadic memory trace and the postsaccadic visual signal belong to the same or to different objects, the model can decide whether these two signals should be integrated or not. Our theory proposes that the necessary input to that model, which is the transsaccadic memory trace, is created by predictive coding.

Future research taking advantage of the blanking paradigm will help to better understand transsaccadic mechanisms, for example by determining under which spatio-temporal conditions and for which stimulus attributes (e.g., color, size, spatial frequency) feature transfer can occur.

We found that briefly blanking a contrast-defined object allows to recover a detailed representation of its orientation. We propose that the blank reveals a phantom-like featural memory trace allowing participants to accurately judge an orientation change across saccades.

\section{Material and methods}

Participants. Twenty-eight participants (mean age 25.93, range 20-36; 12 females) took part in the study, twelve participants per experiment (Exp. 1-2-3). Eight participants participated in two experiments, i.e., two participants performed Exps. 1 and 2; two different participants performed Exps. 1 and 3, and four different participants participated in Exps 2 and 3. Except from one experimenter, they were naïve to the aim of the experiments and were compensated $10 €$ per hour. This experiment was approved by the Ethics Committee of the Faculty for Psychology and Pedagogics of the Ludwig-Maximilians Universität München (approval number 13_b_2015) and conducted in accordance with the Declaration of Helsinki. All participants gave written informed consent.

Apparatus. Participants sat in a quiet and dimly illuminated room. Chin and forehead rests were used to minimize head movements. The experiment was controlled by a PC computer. Gaze position of the dominant eye was recorded using a tower mounted EyeLink 1000 (SR Research Ltd., Ontario, Canada) at a sampling rate of $1000 \mathrm{~Hz}$. The experimental software controlling the display and the response collection as well as eye tracking was implemented in Matlab (The MathWorks, Natick, MA) using the Psychophysics Toolbox ${ }^{53,54}$ and EyeLink Toolbox ${ }^{55}$. Stimuli were presented at a viewing distance of $60 \mathrm{~cm}$, on a VIEWPixx LCD monitor (515 by $290 \mathrm{~mm}$, VPixx Technologies Inc., Saint-Bruno, Canada) with a spatial resolution of 1,920 by 1,080 pixels and a vertical refresh rate of $120 \mathrm{~Hz}$. The monitor luminance was linearized with a Minolta CS-100 luminance meter and a custom software (Minolta, Osaka, Japan). Participants' responses were recorded via a standard keyboard and mouse.

Orientation discrimination tasks. Each trial began with participants looking at the fixation point at the screen center (Fig. 1A). The fixation point was a "bull's eye" with a radius of 0.4 degrees of visual angle (dva), composed of superimposed black $(\sim 0 \mathrm{~cd} / \mathrm{m})$ and white $\left(\sim 57 \mathrm{~cd} / \mathrm{m}^{2}\right)$ disks. When the participant's gaze was detected within a 2.0 dva radius of a virtual circle centered on the fixation point, for at least $200 \mathrm{~ms}$, the trial began with a fixation period randomly between 400 and $700 \mathrm{~ms}$. After this period, the presaccadic grating was presented at a distance of $8.0 \mathrm{dva}$ to the left or to the right of the screen center. Participants were instructed to move their eyes as quickly and as accurately as possible towards the grating center. We detected online the saccade onset as the time at which the gaze position left the $2.0 \mathrm{dva}$ radius virtual circle around the fixation point. We next presented the postsaccadic grating either immediately (No-blank condition, half of the trials; Fig. 1B, upper panel) or after a blank gray screen (Blank condition, half of the trials; Fig. 1B, lower panel) after the saccade onset detection. The average blank durations for Exp. 1, 2 and 3 were 168.42, 170.70 and $164.46 \mathrm{~ms}$, respectively. The saccade onset also triggered the disappearance of the fixation bull's eye. The postsaccadic grating was presented for $300 \mathrm{~ms}$ with a different orientation than the presaccadic grating. Pre- and postsaccadic gratings were green and gray square-wave gratings of 3.2 cycles/dva contained within a circular Gaussian envelope ( $\mathrm{SD}=3.0 \mathrm{dva})$. The background was gray and of a different luminance across experiments (Exp. $1: 15 \mathrm{~cd} / \mathrm{m}^{2} ;$ Exp. $2: 8 \mathrm{~cd} / \mathrm{m}^{2}$; Exp. 3: $15 \mathrm{~cd} / \mathrm{m}^{2}$ ). The gray stripes of the grating were of the same color as the background. The green stripes of the gratings could either be iso- or anisoluminant relative to the background. The isoluminance of the grating was determined for each participant individually (see "Isoluminance assessment" below). Anisoluminant gratings were having a twofold $\left(\sim 30 \mathrm{~cd} / \mathrm{m}^{2}\right.$, Exp.1) or fivefold $\left(\sim 40 \mathrm{~cd} / \mathrm{m}^{2}\right.$, Exp.2) higher luminance as compared to the isoluminant, adjusted value, or being modulated by $6,12,25$ or $50 \%$ as compared to it (Exp. 3). The angle of the presaccadic grating was chosen randomly around the vertical orientation $\left(0^{\circ}\right)$ between $\pm 64^{\circ}, \pm 54.5^{\circ}, \pm 45$ $\circ, \pm 35.5^{\circ}, \pm 26^{\circ}$ (positive and negative angles refer to clockwise and counterclockwise angles, respectively). The postsaccadic grating was randomly rotated by $\pm 1^{\circ}, \pm 4^{\circ}, \pm 7^{\circ}, \pm 10^{\circ}, \pm 13^{\circ}$ or $\pm 16^{\circ}$ relative to the presaccadic grating angle. Participants reported the change in orientation angle of the postsaccadic grating relative to the presaccadic one. To do so, they used the left or the right direction arrow keyboard buttons, corresponding to clockwise or counterclockwise orientation changes, respectively. Responses were followed by a negative-feedback sound in the case of an incorrect response. In Exp. 1 and 2, presaccadic and postsaccadic gratings were either isoluminant or anisoluminant relative to the background, resulting in four distinct combinations of stimuli across saccades (Fig. 2A). In Exp. 3, the pre- and postsaccadic gratings were always of the same luminance in a given trial.

Correct fixation resulted from gaze being maintained within a $2.0^{\circ}$ radius virtual circle centered on the fixation target. Correct saccades resulted from saccades landing within a $2.0^{\circ}$ radius virtual circle centered on the cue. Trials in which, participants incorrectly fixated, made an incorrect saccade, executed their saccades before than $50 \mathrm{~ms}$ or than $350 \mathrm{~ms}$ after the presaccadic grating onset or, blinked were repeated. On average $171.53 \pm 14.60$ trials per participant $(11.91 \pm 1.01 \%)$ were repeated according to these criteria (Exp. $1: 116.50$ trials $=11.42 \%$; Exp. 2: 199.58 trials $=13.86 \%$; Exp. 3: 150.50 trials $=10.45 \%)$. 
Isoluminance assessment. Before each experiment, participants performed a block of 40 trials, in which, using the heterochromatic flicker photometry method ${ }^{56}$, we evaluated individual isoluminance thresholds of the green stripes of the grating relative to the background. The method consists of presenting the grating with a polarity reversal $\left( \pm 180^{\circ}\right.$ phase change) at a frequency of $30 \mathrm{~Hz}$ to produce a strong perceived stimulus flicker as long as the luminance of the grating green stripes doesn't match perceptually with the background luminance. Participants were instructed to minimize the perceived flicker using a computer mouse to adjust the luminance of the green stripes relative to the background. When the flicker was perceived as minimal, they were instructed to press the keyboard space bar. The minimum and the maximum adjustable luminance of the grating green stripes were set between 5 and $25 \mathrm{~cd} / \mathrm{m}^{2}$ (Exp. 1 and Exp. 3) and between 3 and $13 \mathrm{~cd} / \mathrm{m}^{2}$ (Exp. 2). The grating was presented in half of the trials at the screen center that participants fixated. On the other half of the trials the grating was shown 8.0 dva to the left or right of the screen center and participants fixated the central bull's eye. For each participant, a foveal and peripheral threshold of subjective isoluminance was obtained. On average, foveal and peripheral thresholds were $15.47 \pm 0.28 \mathrm{~cd} / \mathrm{m}^{2}$ and $15.83 \pm 0.25 \mathrm{~cd} / \mathrm{m}^{2}$ (Exp. 1), $8.91 \pm 0.42 \mathrm{~cd} / \mathrm{m}^{2}$ and $8.89 \pm 0.45 \mathrm{~cd} / \mathrm{m}^{2}$ (Exp. 2), and, $15.00 \pm 0.29 \mathrm{~cd} / \mathrm{m}^{2}$ and $15.17 \pm 0.39 \mathrm{~cd} / \mathrm{m}^{2}$ (Exp. 3), respectively.

Procedure. The experiments started with one block of the isoluminance assessment followed by eight blocks of the orientation discrimination tasks (Exp. 1, 2 and 3) ran in a single experimental session of about $100 \mathrm{~min}$ (including breaks). In total, each participant performed 1440 correct trials in these eight blocks of 180 trials. In summary, participants performed 15 trials $\times 6$ orientation angles $\times 2$ saccade locations $\times 2$ blank conditions (Blank, No-blank) $\times 4$ stimuli luminance conditions, i.e., Exp.1 and 2: aniso-aniso, iso-iso, aniso-iso, iso-aniso; Exp. 3: 6, 12, 25 and 50\% luminance modulations. All these conditions were randomized within each block.

Data pre-processing. Before proceeding to the analysis of the behavioral results, we scanned the recorded eye-position data offline. Saccades were detected based on their velocity distribution using a moving average over twenty subsequent eye position samples ${ }^{57}$. Saccade onset and offset were detected when the velocity exceeded and fell behind the median of the moving average by 3 SDs for at least $20 \mathrm{~ms}$. We included trials where a correct fixation was maintained within a $2.0^{\circ}$ radius centered on the fixation target, where a correct saccade started at the fixation point and landed within a $2.0^{\circ}$ radius centered on gratings. With such analysis we found that the presaccadic grating was extinguished and replaced by the postsaccadic grating or a blank on average $19.63 \pm 0.14 \mathrm{~ms}$ after the saccade onset and $29.75 \pm 0.75 \mathrm{~ms}$ before the saccade offset. In total, we included on average per participants 1388,1412 and 1229 trials $(98.39 \%, 98.06 \%$ and $85.35 \%$ of the online selected trials, i.e., $86.29 \%, 86.12 \%$ and $77.33 \%$ of all trials played) of the orientation discrimination task of the Experiment 1, 2 and 3, respectively.

Behavioral analysis. The data for the leftward and rightward saccades were collapsed and percentages of correct responses were calculated for each of the seven different angle changes used across all the presaccadic orientations. Subsequently, cumulative Gaussian functions were fitted to these data using the Palamedes toolbox ${ }^{58}$ and the $75 \%$ correct discrimination thresholds were determined for each condition and participant. In each experiment, the eight conditions were normally distributed according to one sample Kolmogorov-Smirnov tests $(0.26>K S s>0.11 ; 0.99>p s>0.32)$. We computed a blanking effect ratio by dividing the orientation threshold observed within trials in which we presented a blank to those observed in trials without a blank and changing this ratio in an improvement percentage, i.e., 100 - $\left(\mathrm{x}_{\text {Blank }}{ }^{\star} 100 / \mathrm{x}_{\text {No-blank }}\right)$. To estimate the overall participant report bias and just noticeable differences (JNDs), we fitted cumulative Gaussian functions to the proportion of clockwise report as a function of the orientation angle change between the pre- and postsaccadic grating. Please note that negative values correspond to counterclockwise angle changes. JND angles correspond to the difference between the orientation change needed to obtain $75 \%$ and $25 \%$ of clockwise reports. Bias angles correspond to the point of subjective equality (PSE), that is the orientation change that would lead to $50 \%$ of clockwise reports.

To calculate the statistical significance of paired comparisons we used a bootstrap method inspired by the bootstrap hypothesis testing ${ }^{59}$. For a given comparison, we firstly drawn 10,000 bootstrap samples from the original pair of compared values (with replacement). Then, we calculated the difference of these two bootstrapped distributions and a test statistic value from that difference distribution (mean / standard error). Finally, we compared the test statistic value to a normal distribution to obtain a two-tailed $p$-value.

Received: 26 November 2019; Accepted: 15 October 2020

Published online: 29 October 2020

\section{References}

1. Østerberg, G. Topography of the layer of rods and cones in the human retina. Acta Ophthalmol. 13, 11-97 (1935).

2. Anstis, S. M. A chart demonstrating variations in acuity with retinal position. Vis. Res. 14, 589-592 (1974).

3. Li, H.-H., Barbot, A. \& Carrasco, M. Saccade preparation reshapes sensory tuning. Curr. Biol. 26, 1564-1570 (2016).

4. Herwig, A. Transsaccadic integration and perceptual continuity. J. Vis. 15, 7 (2015).

5. Rolfs, M. \& Szinte, M. Remapping attention pointers: linking physiology and behavior. Trends Cogn. Sci. 20, 399-401 (2016).

6. Rolfs, M. \& Carrasco, M. Rapid simultaneous enhancement of visual sensitivity and perceived contrast during saccade preparation. J. Neurosci. 32, 13744-13752a (2012).

7. Ohl, S., Kuper, C. \& Rolfs, M. Selective enhancement of orientation tuning before saccades. J. Vis. 17, 1-11 (2017).

8. Cavanagh, P., Hunt, A. R., Afraz, A. \& Rolfs, M. Visual stability based on remapping of attention pointers. Trends Cogn. Sci. https ://doi.org/10.1016/j.tics.2010.01.007 (2010)

9. Melcher, D. \& Colby, C. L. Trans-saccadic perception. Trends Cogn. Sci. https://doi.org/10.1016/j.tics.2008.09.003 (2008).

10. Rayner, K., McConkie, G. W. \& Zola, D. Integrating information across eye movements. Cogn. Psychol. 12, 206-226 (1980).

11. Irwin, D. E., Yantis, S. \& Jonides, J. Evidence against visual integration across saccadic eye movements. Percept. Psychophys. 34, 49-57 (1983). 
12. O’Regan, J. K. \& Levy-Schoen, A. Integrating visual information from successive fixations: does trans-saccadic fusion exist?. Vis. Res. 23, 765-768 (1983).

13. Rayner, K. \& Pollatsek, A. Is visual information integrated across saccades?. Percept. Psychophys. 34, 39-48 (1983).

14. Bridgeman, B. \& Stark, L. Omnidirectional increase in threshold for image shifts during saccadic eye movements. Percept. Psychophys. 25, 241-243 (1979).

15. Wolf, C. \& Schütz, A. C. Trans-saccadic integration of peripheral and foveal feature information is close to optimal. J. Vis. 15, 1-18 (2015).

16. Ganmor, E., Landy, M. S. \& Simoncelli, E. P. Near-optimal integration of orientation information across saccades. J. Vis. 15, 1-12 (2015).

17. Stewart, E. E. M. \& Schütz, A. C. Attention modulates trans-saccadic integration. Vis. Res. 142, 1-10 (2018).

18. Wittenberg, M., Bremmer, F. \& Wachtler, T. Perceptual evidence for saccadic updating of color stimuli. J. Vis. 8, 9-9 (2008).

19. Oostwoud Wijdenes, L., Marshall, L. \& Bays, P. M. Evidence for optimal integration of visual feature representations across saccades. J. Neurosci. 35, 10146-10153 (2015).

20. Schut, M. J., Van der Stoep, N., Fabius, J. H. \& Van der Stigchel, S. Feature integration is unaffected by saccade landing point, even when saccades land outside of the range of regular oculomotor variance. J. Vis. 18, 6 (2018).

21. Weiß, K., Schneider, W. X. \& Herwig, A. A "blanking effect" for surface features: Transsaccadic spatial-frequency discrimination is improved by postsaccadic blanking. Attention Perception Psychophys. 77, 1500-1506 (2015).

22. Szinte, M., Jonikaitis, D., Rolfs, M., Cavanagh, P. \& Deubel, H. Presaccadic motion integration between current and future retinotopic locations of attended objects. J. Neurophysiol. 116, 1592-1602 (2016).

23. Rolfs, M., Jonikaitis, D., Deubel, H. \& Cavanagh, P. Predictive remapping of attention across eye movements. Nat. Neurosci. https ://doi.org/10.1038/nn.2711 (2010).

24. Duhamel, J. R., Colby, C. L. \& Goldberg, M. E. The updating of the representation of visual space in parietal cortex by intended eye movements. Science 1, 1. https://doi.org/10.1126/science.1553535 (1992).

25. Zirnsak, M., Gerhards, R. G. K., Kiani, R., Lappe, M. \& Hamker, F. H. Anticipatory saccade target processing and the presaccadic transfer of visual features. J. Neurosci. 31, 17887-17891 (2011).

26. Zerr, P. et al. Remapping high-capacity, pre-attentive, fragile sensory memory. Sci. Rep. 7, 15940 (2017).

27. Deubel, H., Schneider, W. X. \& Bridgeman, B. Postsaccadic target blanking prevents saccadic suppression of image displacement. Vis. Res. 36, 985-996 (1996).

28. Gysen, V., Verfaillie, K. \& De Graef, P. The effect of stimulus blanking on the detection of intrasaccadic displacements of translating objects. Vis. Res. 42, 2021-2030 (2002).

29. Matsumiya, K., Sato, M. \& Shioiri, S. Contrast dependence of saccadic blanking and landmark effects. Vis. Res. 129, 1-12 (2016).

30. Takano, S. Displacement detection is suppressed by the post-saccadic stimulus. 2, 1-29

31. Paeye, C., Collins, T. \& Cavanagh, P. Transsaccadic perceptual fusion. J. Vis. 17, 14 (2017).

32. Deubel, H., Schneider, W. X. \& Bridgeman, B. Transsaccadic memory of position and form. Prog. Brain Res. 140, 165-180 (2002).

33. Grzeczkowski, L., van Leeuwen, J., Belopolsky, A. V. \& Deubel, H. Spatiotopic and saccade-specific transsaccadic memory for object detail. J. Vis. 20, 2 (2020).

34. Szinte, M. \& Cavanagh, P. Spatiotopic apparent motion reveals local variations in space constancy. J. Vis. 11, 1-20 (2011).

35. Aagten-Murphy, D. \& Bays, P. M. Functions of memory across saccadic eye movements. in Current topics in behavioral neurosciences 155-183 (Springer, Cham, 2018). https://doi.org/10.1007/7854_2018_66

36. Vetter, P., Edwards, G. \& Muckli, L. Transfer of predictive signals across saccades. 3, 1-10 (2012).

37. Edwards, G., Vetter, P., McGruer, F., Petro, L. S. \& Muckli, L. Predictive feedback to V1 dynamically updates with sensory input. Sci. Rep. 7, 16538 (2017).

38. Rao, R. P. N. \& Ballard, D. H. Predictive coding in the visual cortex: a functional interpretation of some extra-classical receptivefield effects. Nat. Neurosci. 2, 79-87 (1999).

39. Sterzer, P., Frith, C. \& Petrovic, P. Believing is seeing: expectations alter visual awareness. Curr. Biol. 20, 1973 (2010).

40. Grzeczkowski, L., Tartaglia, E. M., Mast, F. W. \& Herzog, M. H. Linking perceptual learning with identical stimuli to imagery perceptual learning. J. Vis. 15, 13 (2015).

41. Sasaki, Y. \& Watanabe, T. The primary visual cortex fills in color. Proc. Natl. Acad. Sci. 101, 18251-18256 (2004).

42. Germeys, F., De Graef, P., Van Eccelpoel, C. \& Verfaillie, K. The visual analog: evidence for a preattentive representation across saccades. J. Vis. 10, 1-28 (2010).

43. Edwards, G., VanRullen, R. \& Cavanagh, P. Decoding trans-saccadic memory. J. Neurosci. 38, 1114-1123 (2018).

44. Huber-Huber, C., Buonocore, A., Dimigen, O., Hickey, C. \& Melcher, D. The peripheral preview effect with faces: combined EEG and eye-tracking suggests multiple stages of trans-saccadic predictive and non-predictive processing. Neuroimage 200, 344-362 (2019).

45. Sommer, M. A. \& Wurtz, R. H. Influence of the thalamus on spatial visual processing in frontal cortex. Nature https://doi. org/10.1038/nature05279 (2006).

46. Walker, M. F., Fitzgibbon, E. J. \& Goldberg, M. E. Neurons in the monkey superior colliculus predict the visual result of impending saccadic eye movements. J. Neurophysiol. https://doi.org/10.1152/jn.1995.73.5.1988 (2017).

47. Armstrong, K. M. \& Moore, T. Rapid enhancement of visual cortical response discriminability by microstimulation of the frontal eye field. Proc. Natl. Acad. Sci. https://doi.org/10.1073/pnas.0701104104 (2007).

48. Subramanian, J. \& Colby, C. L. Shape selectivity and remapping in dorsal stream visual area LIP. J. Neurophysiol. https://doi. org/10.1152/jn.00841.2011 (2013).

49. Yao, T., Treue, S. \& Krishna, B. S. An attention-sensitive memory trace in macaque MT following saccadic eye movements. PLOS Biol. 14, e1002390 (2016).

50. Gardner, J. L., Merriam, E. P., Movshon, J. A. \& Heeger, D. J. Maps of visual space in human occipital cortex are retinotopic not spatiotopic. J. Neurosci. https://doi.org/10.1523/jneurosci.5476-07.2008 (2008).

51. Tas, A. C., Moore, C. M. \& Hollingworth, A. An object-mediated updating account of insensitivity to transsaccadic change. J. Vis. 12, 18-18 (2012)

52. Atsma, J., Maij, F., Koppen, M., Irwin, D. E. \& Medendorp, W. P. Causal inference for spatial constancy across saccades. PLoS Comput. Biol. 12, 1-20 (2016).

53. Brainard, D. H. The psychophysics toolbox. Spat. Vis. 10, 433-436 (1997).

54. Pelli, D. G. The VideoToolbox software for visual psychophysics: transforming numbers into movies. Spat. Vis. 10, 437-442 (1997).

55. Cornelissen, F. W., Peters, E. M. \& Palmer, J. The Eyelink toolbox: eye tracking with MATLAB and the psychophysics toolbox. Behav. Res. Methods Instrum. Comput. 34, 613-617 (2002).

56. Bone, R. A. \& Landrum, J. T. Heterochromatic flicker photometry. Arch. Biochem. Biophys. 430, 137-142 (2004).

57. Engbert, R. \& Mergenthaler, K. Microsaccades are triggered by low retinal image slip. Proc. Natl. Acad. Sci. 103, $7192-7197$ (2006).

58. Prins, N. \& Kingdom, F. A. A. Applying the model-comparison approach to test specific research hypotheses in psychophysical research using the Palamedes toolbox. Front. Psychol. https://doi.org/10.3389/fpsyg.2018.01250 (2018).

59. Efron, B. \& Tibshirani, R. J. An Introduction to the Bootstrap. An Introduction to the Bootstrap https://doi.org/10.1007/978-14899-4541-9 (1993). 


\section{Acknowledgements}

We thank Jasna Martinovic for her useful comments and, Vanessa Fetzer and Dorontina Ismajli for help with data collecting. This work was supported by grants of the Deutsche Forschungsgemeinschaft (DFG) to HD (DE336/6-1) and MS (SZ343/1) and a Marie Skłodowska-Curie Action Individual Fellowship to MS (704537).

\section{Author contributions}

Conceptualization, design, writing, L.G., H.D. and M.S.; Methodology and analysis, L.G. and M.S.; Resources, H.D. and M.S.; Investigation, L.G.

\section{Funding}

Open Access funding enabled and organized by Projekt DEAL.

\section{Competing interests}

The authors declare no competing interests.

\section{Additional information}

Correspondence and requests for materials should be addressed to L.G.

Reprints and permissions information is available at www.nature.com/reprints.

Publisher's note Springer Nature remains neutral with regard to jurisdictional claims in published maps and institutional affiliations.

(c) (i) Open Access This article is licensed under a Creative Commons Attribution 4.0 International License, which permits use, sharing, adaptation, distribution and reproduction in any medium or format, as long as you give appropriate credit to the original author(s) and the source, provide a link to the Creative Commons licence, and indicate if changes were made. The images or other third party material in this article are included in the article's Creative Commons licence, unless indicated otherwise in a credit line to the material. If material is not included in the article's Creative Commons licence and your intended use is not permitted by statutory regulation or exceeds the permitted use, you will need to obtain permission directly from the copyright holder. To view a copy of this licence, visit http://creativecommons.org/licenses/by/4.0/.

(c) The Author(s) 2020 